\title{
EL PROYECTO SUBREGIONAL CONO SUR DE CONTROL Y VIGILANCIA DE LA HIDATIDOSIS
}

\author{
Pilar Irabedra ${ }^{1, a}$, Roberto Salvatella ${ }^{1, b}$
}

\begin{abstract}
RESUMEN
El Proyecto Subregional Cono Sur de Control y Vigilancia de la Hidatidosis: Argentina, Brasil, Chile y Uruguay, es una herramienta conjunta y colaborativa de los países para promover la implantación o el fortalecimiento de los programas de control. Se hace una descripción de los antecedentes, de los aspectos institucionales que regulan su organicidad y funcionamiento y de las líneas de acción definidas en el proyecto técnico operativo. Se destaca los logros obtenidos a través de los Proyectos de Cooperación Técnica entre Países así como el desarrollo de enfoques integrales e innovadores y la formación de recursos humanos de los programas de control. Algunos de los desafíos futuros son: lograr la sustentabilidad del Proyecto, implementar los grupos técnicos de análisis y evaluación a solicitud de los países, mejorar los sistemas de información regionales, continuar las actividades de capacitación y entrenamiento de recursos humanos y la expansión y fortalecimiento de la cooperación técnica entre países.
\end{abstract}

Palabras clave: Equinococosis, Control de enfermedades transmisibles; Cooperación Internacional; América del Sur (fuente: DeCS BIREME).

\section{THE SOUTHERN CONE SUB-REGIONAL PROJECT ON CYSTIC ECHINOCOCOSIS CONTROL AND SURVEILLANCE}

\begin{abstract}
Southern Cone Sub-Regional Project on Cystic Echinococcosis Control and Surveillance: Argentina, Brasil, Chile and Uruguay, is a joint and collaborative tool with the aim of promoting the implementation or the strengthening of programs for disease control. The paper describes the background, the institutional aspects that regulates the structure and functions, as well as the guidelines defined in the technical and operational project. The article emphasize the achievements through Projects of Technical Cooperation among Countries, and the development of integrated and innovative approaches for prevention and control of the disease and training of human resources of the control programs. Some of the challenges are: to achieve the sustainability of the project, implementation of technical groups for analysis and assessment at request of the countries, improvement of the regional information systems, to continue training human resources of the control programs and to expand and strengthen the technical cooperation among countries
\end{abstract}

Key words: Echinococcosis, Communicable disease control, International Cooperation; South America (source: MeSH NLM).

\section{INTRODUCCIÓN}

La equinococosis quística es una enfermedad zoonótica crónica causada por el estado larvario del Echinococcus granulosus, endoparásito perteneciente a la clase Cestoda, familia Taeniidae. Para completar su ciclo de vida requiere de dos hospederos mamíferos. Un hospedero definitivo (carnívoro, principalmente el perro) donde se desarrolla la fase adulta o estrobilar, y un hospedero intermediario donde se desarrolla la fase larvaria o metacestode. El Echinococcus granulosus exhibe una sustancial diversidad genética que tiene implicancias importantes en áreas como el desarrollo y diseño de vacunas, en el diagnóstico así como en la efectividad de los tratamientos ${ }^{(1)}$.
La equinococosis quística (hidatidosis) no es una enfermedad nueva. Desde los tiempos de Hipócrates de Cos (460-380 aC) se tenía conocimiento de esta enfermedad en los seres humanos. En el Primer Congreso Panhelénico de 1901, el profesor Couzis, informó que la equinococosis ya había sido observada por los grandes investigadores de la antigüedad. Los textos de Hipócrates, Areteo y otros médicos de la época, demostraban que se conocía el quiste hidatídico de pulmón, hígado y del peritoneo, la presencia de membranas en la expectoración así como la ruptura de quistes hepáticos en la cavidad peritoneal. Ya habían observado también que estas lesiones eran igualmente encontradas en las vísceras de los animales tales como los ovinos, bovinos y porcinos ${ }^{(2)}$.

Organización Panamericana de la Salud, Montevideo, Uruguay.

a Médico Veterinario, Epidemióloga; ${ }^{\text {b }}$ Médico especialista en Salud Pública. 
La expansión y distribución de la hidatidosis por diferentes continentes, según algunas de las teorías impulsadas en la actualidad señalan a la hidatidosis como una enfermedad originaria de Islandia, que fue llevada a las distintas comarcas por los perros de los balleneros. El Profesor Velarde Pérez Fontana manifestó compartir esta tesis pudiendo verificar su exactitud en los focos del Río de la Plata y de la zona Patagónica, demostrando la existencia en esas zonas de perros en los balleneros y que La presencia de maxilares de ballena marca la pauta de la distribución de la enfermedad hidática ${ }^{(2)}$.

Si bien, desde estas épocas remotas es conocida la hidatidosis y se han realizado esfuerzos para su control y prevención, continúa siendo endémica en gran parte del mundo e incluso, en algunas áreas, actualmente parecería ser emergente o reemergente, incluyendo partes de Asia Central, China, Europa, África del Este y América del Sur ${ }^{(3-5)}$

La equinococosis quística es una enfermedad que afecta principalmente las áreas rurales y centros poblados pequeños con características rurales en los que generalmente el acceso a los servicios de salud de las personas, es menor. En las explotaciones pecuarias de estas áreas, existe la costumbre de alimentar a los canes con vísceras resultado de la faena domiciliaria, lo que asegura el mantenimiento del ciclo biológico del parásito.

En las últimas décadas, se ha producido una importante migración rural urbana en gran parte de los países del Cono Sur de América, por ejemplo en Uruguay, de acuerdo con los censos de población en el año 1860 solo el $45 \%$ de la población residía en zonas urbanas, hacia 1930 el porcentaje urbano se estimaba en 63\%, en 1950 se eleva al $73 \%$ y al presente el $91 \%$ de la población reside en zonas urbanas ${ }^{(6)}$. Los cambios sociodemográficos han impactado en las características epidemiológicas de la enfermedad con el desarrollo de focos endémicos secundarios en poblaciones con alta fragilidad socioeconómica que se asientan en los cinturones de las ciudades y en las cuales existen serias deficiencias de infraestructura sanitaria como el acceso a agua segura para bebida y para riego de huertas.

Por otra parte, la complejidad que representa el control de la equinococosis está dada por múltiples determinantes que influyen sobre la vulnerabilidad y receptividad de los ecosistemas y sobre los diferentes grados de exposición. Determinantes estructurales (pobreza, educación, infraestructura), antropológicos, sistemas de producción, prácticas agrícolas, influyen fuertemente en el cambio de los patrones epidemiológicos y de distribución de la enfermedad debiendo ser adaptadas nuevas estrategias para el control.
Se debe destacar la particularidad de que afecta tanto la salud humana como la economía por su efecto sobre la producción animal, debiendo ser encarada entonces como un serio problema de salud y de agricultura. En términos de carga de enfermedad la equinococosis quística tiene un alto impacto tanto en términos de pérdidas económicas como de DALYS (Disability-Adjusted Life Years). En Argentina, durante el transcurso del período 1987-2005 se perdieron en total 47 407,43 años de vida saludable (AVISA) ${ }^{(7)}$. Tomando como ejemplo las pérdidas estimadas solamente para Perú, han sido calculadas en

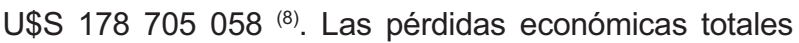
(humanas y animales) estimados para Uruguay fueron de un mínimo de U\$S 2,9 millones al año hasta un máximo de U\$S 22,1 millones anuales ${ }^{(9)}$.

\section{LA HIDATIDOSIS EN EL SUR DE AMÉRICA}

Con relación a su distribución en el continente americano, en Argentina existen tres regiones donde la hidatidosis presenta una elevada tasa de transmisión: la región patagónica al sur del país, la provincia de Buenos Aires y la región del litoral al este del país, en todas ellas asociada con la producción de ovinos. Sin embargo, se encuentra presente en todas las provincias argentinas, incluyendo focos en la región noroeste (en especial las provincias de Tucumán, Salta y Jujuy), región de Cuyo al oeste del país (principalmente el sur de la provincia de Mendoza) y en la región central (provincias de la Pampa, Córdoba y Santa Fe). La tasa de notificación en 2006 fue de 332 casos y en 2007 de 366 casos ${ }^{(10)}$. Los tamizajes ecográficos realizados la provincia de Río Negro en 2007/2008 muestran una incidencia en niños de 6 a 14 años de 0,1\% (11). Se estima en 408083 la cantidad de niños en riesgo de enfermar pertenecientes a 20 jurisdicciones provinciales (7). La prevalencia de infección en fincas ovejeras en la Patagonia (provincias de Río Negro, Chubut, Neuquén, Santa Cruz, Tierra del Fuego y La Pampa), sobre un total de 1042 muestras pertenecientes a 352 fincas ganaderas, se obtuvo un $7,3 \%$ de canes positivos a coproantígeno ${ }^{(12)}$.

En Chile, la hidatidosis es endémica en las regiones donde la ganadería ovina es la principal actividad pecuaria. Presenta una incidencia entre 2 a 2,5/100 000 habitantes; sin embargo, considerando los egresos hospitalarios se estima una incidencia de 10/100 000 (10). La letalidad de la enfermedad alcanza a un $3 \%$ y la mortalidad oscila entre 0,3 a $0,4 / 100000$ habitantes (10). Las regiones australes XI y XII concentran el $60 \%$ de la ganadería ovina, resultando por ende, las que mayores tasas de prevalencia han presentado en los diferentes hospederos. De los egresos hospitalarios del período 2001-2002, el 14,0\% corresponden a menores de 15 años, lo que demuestra que se están produciendo 
infecciones recientes ${ }^{(10)}$. Estudios serológicos puntuales muestran tasas de prevalencia de 51-66/100 000 en la zona norteña de los desiertos y 305-657/100 000 en la zona sureña austral ${ }^{(13)}$. En la región de la Araucanía ha representado más de 1700 egresos hospitalarios en el período 1991-1998, con una media de 13 días de hospitalización afectando a grupos en plena actividad productiva y con una mortalidad importante que provoca daño económico y familiar ${ }^{(14)}$. Datos oficiales registrados en mataderos en la VII Región de Chile revelaron quistes hidatídicos en $13 \%$ de los bovinos, $4,4 \%$ de los ovinos y $4,2 \%$ de los porcinos sacrificados en la Región ${ }^{(15)}$. En el periodo 1990-2000 del total de animales beneficiados se registró un 7,5\% de animales con hidatidosis. Las áreas mas afectadas corresponden a los servicios de salud de Arauco (50,5\%), Araucanía $(25,3 \%)$, Antofagasta $(22,3 \%)$, Osorno $(21,9 \%)$, Aysén $(21,4 \%)$ y Ñuble $(20,8 \%)^{(10)}$.

En el caso de Brasil, en la mayoría de los estados brasileños hay casos esporádicos de hidatidosis. El Estado de Río Grande do Sul, es reconocido como la mas importante área endémica del país. En la década del 80 , una encuesta serológica en humanos en siete municipios de frontera con Uruguay mostró una prevalencia de 8,06/1000 habitantes ${ }^{(10)}$. En el año 1999 fue realizada una nueva encuesta serológica en 18 municipalidades de las áreas endémicas, registrando tasas de prevalencia con un rango de 8,82 a $89,44 / 100000$ hab ${ }^{(16)}$. Datos provenientes de establecimientos de faena en el período 1980-1988 registraron prevalencias de $33,0 \%$ en bovinos y $23,2 \%$ en ovinos ${ }^{(10)}$. En caninos, un estudio realizado en Santana de Livramento resultó en un $27,7 \%$ de canes positivos al test de ELISA coproantígeno en áreas rurales, mientras que no se encontró canes positivos en áreas urbanas y suburbanas ${ }^{(17)}$.

En Uruguay, la enfermedad es endémica en todo el país, estando asociada principalmente con los ciclos perro/ovino. En seres humanos, a través de tamizajes ecográficos se detectan niveles de prevalencia de imágenes quísticas de 1 a $2 \%$ en habitantes de áreas de riesgo (zonas rurales, centros poblados pequeños, áreas de contexto socioeconómico crítico) ${ }^{(18)}$. Un diagnóstico en canes realizado en establecimientos rurales a nivel nacional, mediante el test de ELISA coproantígeno, dio como resultado un $6,4 \%$ de predios con canes positivos y un $4,3 \%$ de perros positivos ${ }^{(19)}$.

Perú, en la actualidad, es probablemente el país de las Américas con una mayor incidencia y prevalencia de equinococosis quística. En seres humanos se han notificado tasas de hasta $79 / 100000$ en provincias como Pasco y de 39/100 000 en Huancavelica (20). Estudios realizados en una ciudad de la costa de Perú, al Sur de
Lima mostraron una incidencia quirúrgica de 32/100.000 para el período 1996-1998. En esta misma área la parasitación con E. granulosus en caninos fue del 6,25\% (21). En la zona de los Andes peruanos se ha reportado prevalencia en humanos de 9,1\%. La prevalencia en ovinos faenados en establecimientos de faena locales en las mismas áreas han sido de hasta $87 \%{ }^{(22)}$.

En Bolivia, la ocurrencia de la enfermedad se caracteriza por altas tasas de incidencia en áreas ganaderas localizadas principalmente en los valles de los departamentos de Potosí, Cochabamba y Tarija. En el altiplano, están en los departamentos de Potosí, Oruro y Cochabamba donde se localiza la mayor parte del rebaño de caprinos, ovinos y camélidos del país.

Desde mediados del siglo $\mathrm{XX}$, los países que integran el Cono Sur de América han implementado diferentes rangos de intervención para enfrentar este problema. Algunos persisten a la fecha, operando regularmente, otros en forma intermitente mientras otros han sido discontinuados. En América, los primeros programas estructurados fueron el Programa Piloto de Estudio y Lucha contra la Hidatidosis, desarrollado por la provincia de Neuquén, Argentina en el departamento de Huilches entre los años 1970 y 1985 y el Programa Piloto de Control de la Hidatidosis en el Departamento de Flores, Uruguay a partir de 1973. Posteriormente Argentina, Chile y Uruguay han contado desde los años 80 con programas de Control. Brasil ha realizado intervenciones limitadas. En Perú se desarrollaron dos Proyectos Piloto entre los años 1975 y 1984 entre varias instituciones: el Ministerio de Salud, la Universidad Nacional Mayor de San Marcos y las Sociedades Agrícolas de Interés Social "Tupac Amarú" y "Pachacutec Ltda.", los que fueron discontinuados.

\section{EL PROYECTO SUBREGIONAL CONO SUR DE CON- TROL Y VIGILANCIA DE LA HIDATIDOSIS: ARGENTI- NA, BRASIL, CHILE Y URUGUAY}

Considerando la situación de los países de la región, surge la necesidad de aplicar una herramienta conjunta y colaborativa, por lo que en el año 2001 los estados miembros que participaron de la RIMSA XII (Reunión Interamericana, a nivel Ministerial, en Salud y Agricultura) crearon el Proyecto Subregional Cono Sur de Control y Vigilancia de la Hidatidosis: Argentina, Brasil, Chile y Uruguay, que cuenta con la Secretaría Técnica de la Organización Panamericana de la Salud (OPS/OMS).

\section{ANTECEDENTES DEL PROYECTO SUBREGIONAL}

EI XIX Congreso Internacional de la Asociación Internacional de Hidatidología realizado en San Carlos 
de Bariloche, Río Negro, Argentina en el año 1999, en el que el Director de la Organización Panamericana de la Salud convocó un grupo de trabajo que revisó y analizó experiencias sobre perspectivas y posibilidades de control y erradicación de la hidatidosis.

La Reunión Interamericana a nivel Ministerial de Salud y Agricultura (RIMSAXII) realizada en Sao Paulo, Brasil en mayo de 2001 en la que se realizaron recomendaciones para que los países continúen fortaleciendo el enfoque de salud pública veterinaria.

La Organización Panamericana de la Salud, en el marco de la resolución 12.R7 RIMSA, designó a la Oficina en Uruguay de la OPS/OMS, como punto focal regional para la cooperación técnica en el control y vigilancia de la hidatidosis.

La Primera Reunión de Jefes de Programas de Hidatidosis convocada por OPS y realizada en el Centro Panamericano de Fiebre Aftosa (PANAFTOSA), Río de Janeiro, Brasil, en octubre de 2001, en la que se elaboró un documento denominado "Plan de acción y estrategias regionales básicas para la eliminación de la hidatidosis humana en América del Sur".

La primera Reunión Constitutiva para el Proyecto Subregional Cono Sur de Control y Vigilancia de la Hidatidosis: Argentina, Brasil, Chile y Uruguay, convocada por el punto focal del Proyecto realizada en Montevideo, Uruguay del 7 al 9 de julio de 2004 y en la cual participaron representantes oficiales de Argentina, Brasil, Chile y Uruguay, representantes de PANAFTOSA, OPS/OMS en Uruguay y expertos de los países de la región y en la cual acordaron solicitar la cooperación técnica de la OPS/OMS para:

1. Establecer las bases institucionales de operación del Proyecto subregional, a través de la creación de una Comisión Intergubernamental que asegure la continuidad gerencial y operacional de las acciones propuestas por la asamblea. La Comisión quedó conformada por los responsables nacionales de los programas de hidatidosis de los países miembro. A la OPS/OMS se le solicitó actuar en la Secretaría Técnica de la Comisión a través de su oficina en Uruguay, con el apoyo de PANAFTOSA y Salud Pública Veterinaria (DPC-VP).

2. Formular un Proyecto de Cooperación Técnica, el que define la organicidad del Proyecto Subregional y da soporte al funcionamiento de la Comisión. Se solicitó contemplar los siguientes requisitos: la Comisión tiene la autoridad para convocar expertos a los efectos de formar grupos científico técnico para sustento de sus actividades, realizar una reunión anual en sedes rotativas, brindar cuando es posible asesoría y capacitación a los países que lo soliciten, promover procesos de evaluación entre los países integrantes del Proyecto, promover la cooperación y coordinación en áreas de frontera y promover la cooperación con otros países endémicos de la Región.

3. Formular un proyecto técnico operacional y metodológico como guía para desarrollar estrategias homólogas en la subregión, cuyos objetivos definidos fueron: promover la consolidación y la extensión de la cobertura de los programas nacionales de control de la hidatidosis en actual ejecución, promover el desarrollo de programas de control en las áreas endémicas aún no cubiertas y promover acciones de coordinación y cooperación en áreas de frontera.

En la 2. ${ }^{a}$ Reunión del Proyecto realizada en la ciudad de Santiago de Chile en el año 2005, fueron aprobados por los Delegados Nacionales los documentos:

- El Proyecto Subregional Cono Sur de Control y Vigilancia de la Hidatidosis: Argentina, Brasil, Chile y Uruguay. Organicidad y Funcionamiento de la Comisión Intergubernamental ${ }^{(23)}$.

- El Proyecto Técnico Operativo del Proyecto Subregional Cono Sur de Control y Vigilancia de la Hidatidosis: Argentina, Brasil, Chile y Uruguay ${ }^{(23)}$.

Las líneas de acción definidas y recogidas en el informe de la segunda reunión realizada en Santiago de Chile fueron:

- Caracterización de las áreas endémicas a través de la recolección de información sistematizada a los efectos de la cuantificación del problema y la definición de la línea inicial.

- Desarrollo de un sistema de vigilancia epidemiológica que permita la evaluación permanente de los factores que condicionan la diseminación de la equinococosis quística y medir el impacto de las medidas de control.

- Adiestramiento de los recursos humanos de acuerdo con la situación epidemiológica local.

- Desarrollo de los servicios de laboratorio para la caracterización de áreas endémicas y el desarrollo de actividades de control.

- Adecuación de los Programas de Educación Sanitaria como componente integral de todos los programas de control.

- Desarrollo de sistemas de apoyo a las actividades de control. Si bien cada país mantiene estrategias específicas, se prevé el desarrollo de instrumentos adecuados para el fortalecimiento de los programas que se inicien o en curso. 


\section{LOGROS Y DESAFÍOS}

El funcionamiento del Proyecto ha mostrado logros significativos para el avance en el control y la prevención de la equinococosis quística.

Se ha realizado seis reuniones anuales con el objetivo de planificar, armonizar, recomendar e instrumentar lineamientos sobre las acciones a realizar en la subregión. Las que han tenido lugar en Montevideo, Uruguay (2004); Santiago, Chile (2005); Porto Alegre, Brasil (2006); Bariloche, Argentina (2007); Coyhaique, Chile (2008) y Colonia, Uruguay (2009).

Se ha contado con la cooperación de la OPS/OMS para la implementación o fortalecimiento de las políticas de prevención y control de la equinococosis quística a través de proyectos de cooperación entre países (Proyectos TCC) los que han sido el soporte para el desarrollo del Proyecto Subregional. Al respecto se puede nombrar:

- La elaboración del "Plan de Prevención y Control de la hidatidosis en el Perú", apoyando a este país en sus capacidades institucionales y técnicas (epidemiológicas y de diagnóstico) - año 2007.

- Definición de los lineamientos del "Plan Integrado de Prevención y Control de la equinococosis quística en el área de frontera Brasil-Uruguay" promoviendo la integración de los servicios locales de salud y fortaleciendo la capacidad técnica por medio de entrenamientos y capacitación de los recursos humanos. - Año 2009.

- El "Proyecto de Erradicación de la Hidatidosis en la Provincia de Tierra del Fuego, Argentina y Región XII, Chile" - año 2007/2008.

- La elaboración del "Plan de Prevención y Control de la equinococosis quística en el Municipio de Tupiza, Bolivia - años 2009/2010.

El Proyecto Subregional ha promovido la revisión de los enfoques integrados en el control de las zoonosis. Es así que áreas destacadas del conocimiento han sido abordadas por expertos en las reuniones técnicas.

Enfoques innovadores como la equinococosis quística dentro del marco de las enfermedades desatendidas, la equinococosis quística como geohelmintiasis y como enfermedad transmitida por alimentos así como el abordaje de los determinantes antropológicos.

Aspectos operativos, de gestión y de comunicación: avances en prevención, vigilancia y control; planes de acción y estrategias regionales básicas; impacto, diagnóstico, manejo y tratamiento de la hidatidosis humana; abordaje de los determinantes epidemiológicos y sociales; estudios de impacto económico y estrategias educativas y de educación sanitaria entre otros temas de interés.

Se ha realizado la publicación de trabajos científicos y de información además de la publicación del Informe de la Reunión Anual con los resúmenes de las presentaciones realizadas por expertos y de Informes de Proyectos de Cooperación Técnica (TCC), realizados en la Región.

Capacitación de los recursos humanos mediante instancias específicas de entrenamiento en las áreas solicitadas por los países: vigilancia, prevención y control; técnicas de diagnóstico laboratorial en huéspedes definitivos e intermediarios; diagnóstico clínico, por imágenes y tratamiento en humanos; vigilancia ambiental; comunicación social.

En el área de laboratorio se ha brindado asistencia técnica mediante el intercambio y transferencia de tecnología.

En líneas generales, se debe destacar que el Proyecto ha cooperado para que los países que lo integran, implementen o fortalezcan actividades de prevención y control de la equinococosis quística esperándose en consecuencia una disminución de su morbi-mortalidad $y$, por lo tanto, una menor carga de enfermedad para las comunidades.

Se ha fortalecido las actividades conjuntas transfronterizas promoviendo la coordinación de los sistemas locales de salud y, por lo tanto, impactando positivamente en el concepto integral de salud.

Dos países de la Región, Argentina y Chile han tomado el primer territorio insular de América del Sur (Tierra del Fuego) en el que ya se han logrado avances tendientes a la erradicación de la equinococosis quística.

La Iniciativa Cono Sur ha logrado el fortalecimiento de la visión conjunta del problema, con la necesidad de juntar e integrar los esfuerzos entre países, apoyados por las agencias de cooperación técnica.

De aquí en más, se plantean importantes desafíos, entre los que se encuentran: la sustentabilidad del Proyecto a los efectos de poder cumplir con el propósito de "promover acciones nacionales e internacionales conducentes a que todos los países de la región afectados por la hidatidosis adopten programas que permitan eliminar la enfermedad en el hombre y controlarla en las especies ganaderas de importancia económica" (23). 
Implementación de grupos técnicos de análisis y evaluación que a solicitud de los países y a través de visitas en terreno puedan brindar las recomendaciones o certificaciones pertinentes; mejora de los sistemas de información regionales normalizando los sistemas de recolección de información; continuar con las actividades de capacitación y adiestramiento de recursos humanos vinculados con los Programas de Control, el logro de la expansión de la cooperación técnica a otras áreas endémicas que lo soliciten así como el fortalecimiento de la Cooperación Técnica entre países.

\section{REFERENCIAS BIBLIOGRÁFICAS}

1. Mc Manus DP, Thompson RC. Molecular epidemiology of cystic echinococcosis. Parasitology. 2003;127(Suppl):S3751.

2. Turnes A. La hidatidosis como problema de salud pública. Una mirada histórica. $1^{\text {a }}$ ed. Montevideo: OPS; 2009.

3. Craig P, Pawlowski Z. Cestode zoonoses: echinococcosis and cysticercosis. An emergent and global problem. NATO Science Series. Vol. 341. Amsterdam: IOS Press; 2001.

4. Larrieu E, Bellotto A, Arámbulo III P, Tamayo H. Echinococcosis quística: epidemiología y control en América del Sur. Parasitol Latinoam. 2004;59(2):82-89.

5. Jenkins DJ, Romig T, Thompson RC. Emergence/reemergence of Echinococcus spp. A global update. Int J Parasitol, 2005;35(11-12):1205-19.

6. Instituto Nacional de Estadística - Uruguay. Tamaño de la población y tasas de crecimiento. Montevideo: INE; 2000.

7. GuarneraE. Hidatidosis enArgentina. Carga deenfermedad. $1^{a}$ ed. Buenos Aires: Organización Panamericana de la Salud; 2009.

8. Pérez CR. Proyecto de control de hidatidosis en el Perú por vigilancia epidemiológica. [Tesis Doctoral]. Lima: Facultad de Medicina, Universidad Nacional Mayor de San Marcos; 2007.

9. Torgerson $\mathbf{P}$, Carmona $\mathbf{C}$, Bonifacino R. Estimating the economic effects of cystic echinococcosis: Uruguay, a developing country with upper-middle income. Ann Trop Med Parasitol. 2004;94(7):703-13.

10. Organización Panamericana de la Salud. Informe del Proyecto Subregional Cono Sur de control y Vigilancia de la Hidatidosis: Argentina, Brasil, Chile y Uruguay. Primera Reunión Constitutiva. Montevideo: OPS; 2004.

11. Larrrieu E, Del Carpio M, Mercapide CH, Salvitti JC, Sustercic J, Moguilensky J, et. al. Programme for ultrasound diagnoses and treatment with albendazol of cystic echinococcosis in asymptomatic carriers: ten years of follow up of carriers. Acta Trop. 2011;117(1):1-5.
12. Cavagion L, Perez A, Santillán G, Zanini F, Jensen O, Saldía L, et. al. Diagnosis of cystic echinococcosis on sheep farms in the south of Argentina: areas with a control program. Vet Parasitol. 2005;128(1-2):73-81.

13. Schenone $H$, Contreras M, Salinas $P$, Sandoval L, Saavedra T, Rojas A. Hidatidosis humana en Chile. Seroprevalencia y estimación del número de personas infectadas. Bol Chil Parasitol. 1999;54(3-4):70-73.

14. Aliaga F, Oberg C. Epidemiología de la hidatidosis humana en la IX Región de la Araucanía, Chile. 1991-1998. Bol Chil Parasitol. 2000;55(3-4):54-58.

15. Apt W, Pérez C, Galdamez E, Campano S, Vega F, Vargas D, et. al. Equinococosis/hidatidosis en la VII Región de Chile: diagnóstico e intervención educativa. Rev Panam Salud Publica. 2000;7(1):8-16.

16. Souza MAT, Mardini LB, Fernandes C, Tiecher F. Diagnóstico sorológico de hidatidose humana na população rural de 18 municípios do Rio Grande do Sul, Brasil. En: XVI Congresso Brasileiro de Parasitologia: Livro de resumos. Poços de Caldas: Sociedade Brasileira de Parasitologia; 1999. p.35.

17. Farias L, Malgor R, Cassaravilla $C$, Bragança $C$, de la Rue M L. Echinococcosis in southern Brazil: efforts toward implementation of a control program in Santana do Livramento, Rio Grande do Sul. Rev Inst Med Trop Sao Paulo. 2004;46(3):153-56.

18. Uruguay, Comisión Nacional de Zoonosis. Plan Estratégico 2006 - 2009. Montevideo: Comisión Nacional de Zoonosis; 2006.

19. Organización Panamericana de Salud. Informe del Proyecto Subregional Cono Sur de Control y Vigilancia de la Hidatidosis: Argentina, Brasil, Chile y Uruguay. Informe de la 3a . Reunión. Porto Alegre: OPS; 2006.

20. Organización Panamericana de Salud. Informe Final del Proyecto TCC de Fortalecimiento de la Cooperación Técnica sobre Hidatidosis entre Uruguay y Perú. Agosto Diciembre 2007. Montevideo: OPS; 2009.

21. Moro PL, Lopera L, Cabrera M, Cabrera G, Silva B, Gilman RH, et al. Short report: endemic focus of cystic echinococcosis in a coastal city of Peru. Am J Trop Med Hyg. 2004;71(3):327-29.

22. Moro PL, Mc Donald J, Gilman RH, Silva B, Verastegui M, Malqui V, et al. Epidemiology of Echinococcus granulosus infection in the central Peruvian Andes. Bull World Health Organ. 1997;75(6):553-61.

23. Organización Panamericana de Salud. Informe del Proyecto Subregional Cono Sur de Control y Vigilancia de la Hidatidosis: Argentina, Brasil, Chile y Uruguay. Segunda Reunión. Santiago de Chile: OPS; 2005.

Correspondencia: Dra. Pilar Irabedra.

Correo electrónico: irabedrp@uru.ops-oms.org 